\section{OUTCOME OF BABIES FROM THE POSTNATAL WARD WHO UNDERWENT ECHOCARDIOGRAPHY}

\section{A. Singh, S.V. Rasiah}

Neonates, Birmingham Women's Foundation NHS Trust, Birmingham, UK

Objective: To review our echocardiography service for postnatal ward babies, focusing on the indications, findings and the subsequent outcomes.

Methods: Information on all echocardiograms performed on the neonatal unit was collected prospectively over 24 months (January 2008 to December 2009). We then retrospectively reviewed all echocardiograms carried on babies from the postnatal ward over the same period.

Results: 285 echocardiograms were performed on 244 babies in the postnatal ward. The most common indication for performing echocardiography was murmur in $52 \%$ babies. The other indications for echocardiography included: $29 \%$ failed pulse oximetry, $8 \%$ family history of congenital heart disease, $3 \%$ antenatal cardiac concerns, $3 \%$ dysmorphology, $3 \%$ dysrhythmia and $2 \%$ weak femoral pulses. The median age for performing the scan was two days. In $40 \%$ babies, the echocardiogram was normal. The lesions identified include; $25 \%$ patent ductus arteriosus, $15 \%$ ventricular septal defects, $9 \%$ patent foramen ovale or atrial septal defect, $4.4 \%$ right ventricular outflow problems, $2.3 \%$ left ventricular outflow problems, $1.5 \%$ tricuspid regurgitation, $1 \%$ atrioventricular septal defect, $0.4 \%$ transposition of great arteries, $0.4 \%$ tetralogy of fallot and $1 \%$ persistent pulmonary hypertension. $41 \%$ babies were discharged with no follow-up. $36 \%$ babies were followed up in the local cardiac murmur clinic and $23 \%$ babies were referred to the paediatric cardiologist.

Conclusion: Postnatal echocardiogram is valuable in early identification and appropriate referral of structural heart defects to the paediatric cardiologist. It has an equally important role in providing reassurance to families of babies with normal hearts prior to discharge from hospital.

\section{8}

\section{COMPARISON OF OUTCOME OF LOCAL AND EXTERNALLY REFERRED MONOCHORIONIC TWIN PREGNANCIES}

\author{
K. Ali ${ }^{1}$, S. Lang ${ }^{1}$, P. Muthukumar ${ }^{1}$, A. Curley ${ }^{1}$, \\ J. Brocklesby' ${ }^{2}$ E. Murdoch \\ ${ }^{1}$ Neonatal Unit, ${ }^{2}$ Fetal-Maternal Medicine, \\ Cambridge University Hospital NHS Foundation \\ Trust, Cambridge, UK
}

Aim: To assess the fetal and neonatal outcome of monochorionic pregnancies in a tertiary referral centre, comparing local women and those referred from external units.

Background: The unit policy is to deliver monochorionic diamniotic twins (MCDA) at 36 weeks and monchorionic monoamniotic twins (MCMA) at 32 weeks by ELCS. Network referrals deliver at their local hospital unless otherwise indicated.

Method: Data from a 3 year audit of MCDA and MCMA twin pregnancies referred to the fetal medicine unit (FMU) was collected. Fetal medicine reports and medical records were analysed.

Results: There were a total of 80 referrals to the Fetal Medicine Unit (FMU); 47 local and 33 from the network. The median gestation at referral was 13 weeks. There were 28 deliveries at 36 weeks by elective $\mathrm{C} / \mathrm{S}$ and 33 deliveries before 36 weeks by emergency $\mathrm{C} / \mathrm{S}$. Antenatal steroids were given to $93 \%$ of the cases at a median gestation of 24 weeks. There were 17 cases of TTTS, 4 referrals for laser therapy and 6 for amnio reduction. Rates of complications and requirement for intensive care were significantly greater among infants referred antenatally from the network hospitals.

Conclusions: Case series of outcomes from tertiary referral centres are likely to be biased due to higher rates of complications among pregnancies referred from external units. 